\title{
The spectrum of an asymptotically hyperbolic Einstein manifold
}

\author{
JOHN M. LEE ${ }^{1}$
}

\begin{abstract}
This paper relates the spectrum of the scalar Laplacian of an asymptotically hyperbolic Einstein metric to the conformal geometry of its "ideal boundary" at infinity. It follows from work of R. Mazzeo that the essential spectrum of such a metric on an $(n+1)$-dimensional manifold is the ray $\left[n^{2} / 4, \infty\right)$, with no embedded eigenvalues; however, in general there may be discrete eigenvalues below the continuous spectrum. The main result of this paper is that, if the Yamabe invariant of the conformal structure on the boundary is non-negative, then there are no such eigenvalues. This generalizes results of R. Schoen, S.-T. Yau, and D. Sullivan for the case of hyperbolic manifolds.
\end{abstract}

\section{Introduction.}

One of the most important global invariants of a complete, non-compact Riemannian manifold $(M, g)$ is the infimum of the $L^{2}$ spectrum of its Laplacian $\Delta_{g}=d^{*} d$, denoted $\lambda_{0}(g)$. In spite of its importance and the simplicity of its definition, $\lambda_{0}(g)$ is notoriously hard to compute except in simple examples. It reflects in a subtle way the interaction among the topology of the manifold, the curvature of the metric, and the asymptotic behavior of curvature and volume at infinity.

Much more is known about $\lambda_{0}(g)$ for hyperbolic manifolds (Riemannian manifolds with constant sectional curvature equal to -1 ). For example, on $(n+1)$-dimensional hyperbolic space the Laplacian has purely continuous spectrum, consisting of the ray $\left[n^{2} / 4, \infty\right)$, so $\lambda_{0}(g)=n^{2} / 4$. Complete hyperbolic manifolds, which are quotients of the unit ball $B^{n+1}$ by Kleinian groups, can in general have both discrete and continuous spectra. In particular, for a hyperbolic manifold $\left(B^{n+1} / \Gamma, g\right)$ that is geometrically finite (i.e. $\Gamma$

\footnotetext{
${ }^{1}$ Research supported in part by National Science Foundation grant DMS 9101832.
} 
has a fundamental domain bounded by finitely many geodesic hyperplanes), the continuous spectrum is still $\left[n^{2} / 4, \infty\right)$, but there may be finitely many discrete eigenvalues below $n^{2} / 4$ [LP]. In this case, the key to understanding $\lambda_{0}(g)$ is the limit set $\Lambda(\Gamma)$, which is by definition the set of limit points in the sphere $S^{n}=\partial B^{n+1}$ of the action of $\Gamma$ on $\overline{B^{n+1}}$ : in [Su], D. Sullivan showed that if $\left(B^{n+1} / \Gamma, g\right)$ is geometrically finite, there are eigenvalues below $n^{2} / 4$ if and only if $\Lambda(\Gamma)$ has Hausdorff dimension $d>n / 2$, and in that case $\lambda_{0}(g)=d(n-d)$.

On the other hand, recent work of R. Schoen and S.-T. Yau on conformally flat manifolds sheds light on $\Lambda(\Gamma)$ from a different direction. When $M=B^{n+1} / \Gamma$ is geometrically finite and without cusps, the action of $\Gamma$ on $B^{n+1}$ extends to a free and properly discontinuous action on the open subset $\Omega=S^{n} \backslash \Lambda(\Gamma)$ of the sphere; the quotient $S=\Omega / \Gamma$ is the "ideal boundary" of $M$, and carries a natural conformal structure $[\widehat{g}]$ which is locally conformally flat. In [SY], Schoen and Yau studied locally conformally flat manifolds with the aim of understanding the behavior of the Yamabe invariant $y[\widehat{g}]$, which is the infimum of the total scalar curvature functional $\int_{S} R_{\tilde{g}} d V_{\tilde{g}}$ over unit-volume metrics in the conformal class [ $\left.\widehat{g}\right]$. In particular, they were able to characterize those locally conformally flat structures with non-negative Yamabe invariant: they showed that a compact, locally conformally flat manifold $(S,[\widehat{g}])$ with $y[\widehat{g}] \geq 0$ can always be realized as a quotient of a simply-connected domain $\Omega \subset S^{n}$ by a Kleinian group; and among such quotients, the ones with $y[\widehat{g}] \geq 0$ are exactly those for which the limit set has Hausdorff dimension $d \leq(n-2) / 2$.

Combining the implications $y[\hat{g}] \geq 0 \Longrightarrow d \leq(n-2) / 2 \Longrightarrow d \leq$ $n / 2 \Longrightarrow \lambda_{0}(g)=n^{2} / 4$ yields a direct relation between the global Riemannian geometry of a hyperbolic manifold and the global conformal geometry its ideal boundary: if $(M, g)$ is a complete, non-compact, geometrically finite, $(n+1)$-dimensional hyperbolic manifold without cusps, and the conformal structure on its ideal boundary has non-negative Yamabe invariant, then $\lambda_{0}(g)=n^{2} / 4$.

The purpose of this paper is to extend this result to a much more general class of Riemannian and conformal structures, where there is no group action to mediate between the two structures. Just as many locally conformally flat manifolds can be realized as ideal boundaries of complete hyperbolic manifolds, many compact conformal manifolds can be realized as "conformal infinities" of complete, asymptotically hyperbolic Einstein metrics. Suppose $\bar{M}$ is a compact manifold-with-boundary, and $g$ is a complete Riemannian metric on the interior $M$ of $\bar{M}$. One says that $g$ is conformally compact if, for any smooth defining function $\rho$ (i.e. a smooth function on $\bar{M}$ that is positive 
in $M$ and vanishes to first order on $\partial M), \bar{g}=\rho^{2} g$ extends continuously to a positive definite metric on $\bar{M}$; it is conformally compact of order $C^{m, \alpha}$ if $\bar{g} \in C^{m, \alpha}(\bar{M})$. The conformal class of the boundary metric $\widehat{g}=\left.\bar{g}\right|_{T \partial M}$ is invariantly defined, and is called the conformal infinity of $g$. For example, if $B^{n+1} / \Gamma$ is a geometrically finite hyperbolic manifold without cusps, then its hyperbolic metric is conformally compact, and its conformal infinity is the induced locally conformally flat structure on the boundary manifold $\Omega / \Gamma$.

If $(M, g)$ is conformally compact of order at least $C^{2,0}$ and $\rho$ is any defining function, a straightforward computation as in [M1] shows that the sectional curvatures of $g$ approach $-|d \rho|_{\bar{g}}^{2}$ at $\partial M$. Accordingly, one says $g$ is asymptotically hyperbolic of order $C^{m, \alpha}$ if $g$ is conformally compact of order $C^{m, \alpha}, m+\alpha \geq 2$, and $|d \rho|_{\bar{g}}^{2}=1$ along $\partial M$.

In [GL], C. R. Graham and I showed that every conformal structure on $S^{n}$ which is sufficiently close to that of the round metric is the conformal infinity of an Einstein metric on $B^{n+1}$ close to the hyperbolic one. More recently [L], I generalized this result to conformal structures close to the conformal infinity of any negatively curved, asymptotically hyperbolic Einstein metric. It can be shown that these Einstein metrics are unique within the class of metrics sufficiently close to the original Einstein metric; they may very well be globally unique. Thus at the very least, a large open subset of the conformal classes on the sphere have concrete realizations as conformal infinities of Einstein metrics, and to date no one has found any obstructions to a conformal class on a boundary having such a realization.

It has been shown by R. Mazzeo [M3] that the continuous spectrum of the Laplacian on an asymptotically hyperbolic manifold consists of the ray $\left[n^{2} / 4, \infty\right)$ with no embedded eigenvalues, just as in the hyperbolic case; however, in general there may be finitely many eigenvalues in the interval $\left(0, n^{2} / 4\right)$. When the asymptotically hyperbolic metric is Einstein, one might expect a relationship between its spectrum and global conformal invariants of its conformal infinity. This expectation is justified by the following theorem, which is the main result of this paper.

Theorem A. Let $\bar{M}$ be a compact $(n+1)$-dimensional manifold-with-boundary. Suppose $g$ is a smooth Einstein metric on $M=\operatorname{Int} \bar{M}$, which is asymptotically hyperbolic of order $C^{3, \alpha}$ and has smooth conformal infinity [ $\widehat{g}$ ]. If $y[\widehat{g}] \geq 0$, then $\lambda_{0}(g)=n^{2} / 4$; in particular, $\Delta_{g}$ has no $L^{2}$ eigenfunctions.

This is proved by constructing a positive function $\varphi$ satisfying $\left(\Delta_{g}-\right.$ $\left.n^{2} / 4\right) \varphi \geq 0$, since the existence of a positive supersolution to $\Delta_{g}-\lambda$ implies $\lambda \leq \lambda_{0}(g)[\mathrm{CY}]$. The strategy for finding a suitable test function $\varphi$ is 
first to prove the existence of positive solutions to the eigenvalue equation $\left(\Delta_{g}+n+1\right) u=0$ with prescribed growth at infinity, and then to show that, for an appropriate choice of asymptotic growth, a power of $u$ satisfies the desired estimate. The hardest step is a gradient estimate for $u$ of the form $|d u|_{g}^{2} \leq u^{2}$. This estimate proceeds in two steps. First we show that the Einstein condition implies that $|d u|_{g}^{2}-u^{2}$ is subharmonic. Then, by analyzing the asymptotics of $u$ near the boundary, we show that $|d u|_{g}^{2}-u^{2}$ has a continuous extension to the boundary, and, under the assumptions that $g$ is Einstein and $y[\widehat{g}] \geq 0$, can be made non-positive there by a judicious choice of $u$.

It should be mentioned that the technical details of the asymptotic analysis are made somewhat more complicated by the fact that we are assuming only rather weak regularity for $\bar{g}$; if one knew that $\bar{g}$ were smooth up to $\partial M$, one could work in Fermi coordinates for $\bar{g}$ and the argument would be a little more straightforward. However, from asymptotic computations (cf. [FG]) we know that smoothness has to fail generically in even dimensions, since a logarithmic term appears in the asymptotic expansion for the Einstein equation. The Einstein metrics shown to exist in $[\mathrm{L}]$ all have at least $C^{3, \alpha}$ regularity when the conformal infinity is sufficiently regular, so Theorem A applies to them. It is reasonable to conjecture that any asymptotically hyperbolic Einstein metric with smooth conformal infinity has (in suitable coordinates) an asymptotic expansion in powers of $\rho$ and $\log \rho$; since the expansion is regular up to a term of the form $\rho^{n} \log \rho$ in even dimensions and to all orders in odd dimensions, the regularity hypothesis of Theorem A would always be satisfied.

Already in the hyperbolic case one can see that Theorem A is not sharp: using the results of Sullivan and Schoen/Yau quoted above, one need only construct an appropriate Kleinian group whose limit set has dimension $d$ satisfying $(n-2) / 2<d \leq n / 2$ to obtain an example in which $y[\widehat{g}]<0$ but still $\lambda_{0}(g)=n^{2} / 4$. Many such examples are easily obtained by considering compact $(d+1)$-dimensional hyperbolic manifolds $B^{d+1} / \Gamma$, where $d$ is the integer part of $n / 2$, and extending $\Gamma$ to act on $B^{n+1}$ in the obvious way; in this case the limit set is all of the equatorial $d$-sphere.

In these examples, the $L^{2}$ eigenfunctions seem to arise in some sense from the topology of $M=B^{n+1} / \Gamma$, reflected in the size of the group $\Gamma$. One might be tempted to conjecture that the converse to Theorem $\mathrm{A}$ is true at least for conformally compact Einstein metrics on the ball. However, this is also apparently not the case. H. Pedersen [P] constructed a family of asymptotically hyperbolic Einstein metrics on $B^{4}$ depending on a real parameter $t$, whose conformal infinities are the Berger metrics $\widehat{g}_{t}=\sigma_{1}^{2}+$ 
$\sigma_{2}^{2}+t \sigma_{3}^{2}$ (written in terms of the standard left-invariant coframe $\left\{\sigma_{j}\right\}$ for $\left.S^{3}=S U(2)\right)$. As $t \rightarrow+\infty, y\left[\widehat{g}_{t}\right]$ decreases to $-\infty$, while the Einstein metrics converge uniformly on compact sets to the complex hyperbolic metric, so $\lambda_{0}\left(g_{t}\right) \searrow 2$. Numerical studies performed by my student John Roth suggest that, as $t$ increases, the eigenvalues below $n^{2} / 4=9 / 4$ first appear some time after $y\left[\widehat{g}_{t}\right]$ becomes negative, so there is a range of values of $t$ for which the converse to Theorem A is violated.

There are various ways in which Theorem A could be sharpened: for example, by finding a necessary and sufficient condition on $[\widehat{g}]$ for $\lambda_{0}(g)$ to be equal to $n^{2} / 4$, or, even better, by finding a formula for $\lambda_{0}(g)$ in terms of conformal invariants of $[\widehat{g}]$. Similarly, one might hope for a formula for $y[\widehat{g}]$ in terms of Riemannian invariants of $g$, or at least a necessary and sufficient condition on $g$ for $y[\widehat{g}] \geq 0$. A useful first step would be to find a reasonable generalization of the Hausdorff dimension of $\Lambda(\Gamma)$ that makes sense for arbitrary conformal structures, or at least for those that are conformal infinities of Einstein metrics.

The outline of the paper is as follows: $\S 2$ presents basic definitions; $\S 3$ develops the analytic results about the Laplacian on asymptotically hyperbolic manifolds that are needed to solve $\left(\Delta_{g}+n+1\right) u=0$; $\S 4$ shows how to use a solution $u$ to construct a supersolution to $\Delta_{g}-n^{2} / 4$, and shows that $|d u|_{g}^{2}-u^{2}$ is subharmonic; and in $\S 5$ the asymptotic expansion of $u$ is computed and used to show that $|d u|_{g}^{2}-u^{2} \leq 0$ on the boundary. The Appendix is devoted to a proof of an elementary lemma regarding Hölder regularity of solutions to ODE's that I was unable to find in the literature.

I am indebted to Rafe Mazzeo for a number of useful conversations regarding the ideas in this paper, most particularly for introducing me to the literature on hyperbolic manifolds and for making a crucial suggestion regarding the ODE lemma in the Appendix.

\section{Definitions.}

In this section we define our terms, and introduce the metrics and function spaces we will be be working with. Throughout this paper, smooth will always mean $C^{\infty}$.

Let $\bar{M}$ be a smooth, compact, $(n+1)$-dimensional manifold-with-boundary. For technical reasons that will appear in the proof of Proposition 3.3 below, it is convenient to assume in general that $\partial M$ is the union of two disjoint closed submanifolds, labeled $\partial_{0} M$ and $\partial_{\infty} M$, with $\partial_{\infty} M$ non-empty. We will write $M=\bar{M} \backslash \partial_{\infty} M$, which is a non-compact manifold with boundary. We call $\partial_{0} M$ the inner boundary of $M$ and $\partial_{\infty} M$ its boundary at infinity. By 
background coordinates for $M$, we will always mean a smooth coordinate chart for an open subset of $\bar{M}$.

A defining function for $M$ will be a real-valued function $\rho \in C^{1}(\bar{M})$ which vanishes to first order on $\partial_{\infty} M$ and is positive elsewhere. A smooth defining function will always mean a defining function that is smooth on $\bar{M}$.

As in the introduction, a smooth metric $g$ on $M$ is said to be conformally compact if, for some (hence any) smooth defining function $\rho$, the tensor $\bar{g}=\rho^{2} g$ has a continuous extension to $\bar{M}$, and is positive definite there. We say $g$ is conformally compact of order $C^{m, \alpha}$ if $\bar{g} \in C^{m, \alpha}(\bar{M})$. (Note that $g$ is always smooth up to the inner boundary.) We say $g$ has smooth conformal infinity if the induced boundary metric $\widehat{g}=\left.\bar{g}\right|_{T \partial_{\infty} M}$ on $\partial_{\infty} M$ is smooth for any smooth defining function $\rho$.

Let $u$ be a function which is $m$-times continuously differentiable on $M$. For $s \in \mathbf{R}$ and $0 \leq \alpha \leq 1$, we will define weighted Hölder norms $\|u\|_{m, \alpha}^{(s)}$ as follows. First, in the special case in which $M$ is a smoothly bounded open subset of $\mathbf{R}^{n+1}$, we define the norms as in [GL]:

$$
\begin{aligned}
\|u\|_{m, 0}^{(s)}:= & \sum_{l=0}^{m} \sum_{|\gamma|=l}\left\|d^{-s+l} \partial^{\gamma} u\right\|_{L^{\infty}} \\
\|u\|_{m, \alpha}^{(s)}:= & \|u\|_{m, 0}^{(s)}+ \\
& +\sum_{|\gamma|=m} \sup _{x, y}\left[\min \left(d_{x}^{-s+m+\alpha}, d_{y}^{-s+m+\alpha}\right) \frac{\left|\partial^{\gamma} u(x)-\partial^{\gamma} u(y)\right|}{|x-y|^{\alpha}}\right],
\end{aligned}
$$

where $d_{x}$ is the Euclidean distance from $x$ to $\partial_{\infty} M$, and for a multi-index $\gamma, \partial^{\gamma}=\partial^{|\gamma|} / \partial x^{\gamma}$. In the more general case of a manifold-with-boundary, the same norms can be defined using a covering by background coordinate charts and a subordinate partition of unity in the usual way.

In either case, let $\Lambda_{m, \alpha}^{s}(M)$ denote the Banach space of $m$-times continuously differentiable functions on $M$ for which $\|u\|_{m, \alpha}^{(s)}$ is finite. We also let $\widetilde{\Lambda}_{m, \alpha}^{s}(M)$ denote the closed subspace of $\Lambda_{m, \alpha}^{s}(M)$ consisting of functions that vanish on $\partial_{0} M$. By [GL, Proposition 3.3(13)] these spaces are independent of choices of coordinates or partition of unity. Near $\partial_{0} M$ these are just functions which are $C^{m, \alpha}$ up to the boundary (and, in the case of the $\widetilde{\Lambda}$ spaces, satisfy homogeneous Dirichlet boundary conditions there), while near $\partial_{\infty} M$ they satisfy degenerate estimates that are weaker than the usual Hölder estimates. The properties of these spaces are summarized in [GL, Prop. 3.3]; one important property we will use repeatedly is the following: 
if $m \geq 1$, for any smooth defining function $\rho$,

$$
\Lambda_{m, \alpha}^{s}(M)=\rho^{s} \Lambda_{m, \alpha}^{0}(M)=\left\{\rho^{s} u: u \in \Lambda_{m, \alpha}^{0}(M)\right\} .
$$

Throughout this paper, we will use the summation convention and classical index notation, with covariant derivatives indicated by indices preceded by a semicolon. Barred quantities will denote covariant derivatives and curvature invariants computed with respect to $\bar{g}$, and unbarred quantities with respect to $g$, with the indicated metric and its inverse used in each case to lower and raise indices. We will usually omit the semicolon for covariant derivatives of a scalar function. For example,

$$
\bar{\rho}^{i}=\bar{g}^{i j} \rho_{j}=\bar{g}^{i j} \partial \rho / \partial x^{j}
$$

are the components of the $\bar{g}$-gradient of $\rho$.

\section{Analytic Preliminaries.}

The purpose of this section is to present some basic analytic facts about the Laplace operator on conformally compact manifolds. These results can be derived, for example, from the parametrix construction for elliptic edge operators due to Rafe Mazzeo [M2]; however, the results we need follow in a straightforward way from the analysis in [GL], so I have chosen to present direct proofs.

Throughout this section $(M, g)$ will be asymptotically hyperbolic of order $C^{k, \beta}, k \geq 1$, possibly with inner boundary; $\rho$ will be a fixed smooth defining function; and $\bar{g}=\rho^{2} g$. Let $\Delta_{g}=d^{*} d$ denote the scalar Laplacian of $g$. By a slight generalization of the arguments in [GL, §3], we obtain the following result:

Lemma 3.1. For a non-negative integer $m$ and real number $0<\alpha<1$ with $m+1+\alpha \leq k+\beta$, let $h \in \Lambda_{m, \alpha}^{0}(M)$ be a real-valued function such that $h \geq \delta>0$ on $M$; and let $X$ be a vector field on $M$ whose coefficients in any background coordinate chart are in $\Lambda_{m, \alpha}^{1}(M)$. Then

$$
\Delta_{g}+X+h: \widetilde{\Lambda}_{m+2, \alpha}^{0}(M) \rightarrow \Lambda_{m, \alpha}^{0}(M)
$$

is an isomorphism.

Proof. In the special case that $X=0, \partial_{0} M=\emptyset$, and $\bar{g}$ is smooth, this follows directly from Propositions 3.4, 3.7, and 3.8 (and the remark following 
Proposition 3.8) in [GL]. The generalization to the present case is straightforward: First, the presence of the first-order term $X$ has no effect on the argument, as one can easily check; in particular, the test function estimate $\left(\Delta_{g}+X+h\right) \varphi \geq \delta \varphi$ still holds with $\varphi=1$, and the arguments of [GL, Prop. 3.8] go through unchanged. Second, the presence of the inner boundary $\partial_{0} M$ can be handled by applying the usual boundary regularity theory and Schauder estimates for uniformly elliptic Dirichlet problems. Finally, if $\bar{g}$ is only in $C^{k, \beta}(\bar{M}) \subset C^{m+1, \alpha}(\bar{M})$, the same argument goes through; the only point that requires attention is to check that the coefficients of the $r$-th order terms in $\Delta_{g}$ are in $\Lambda_{m, \alpha}^{r}(M)$, which is just what is needed to insure that there is a uniform constant $C$ in the estimate of [GL, Prop. 3.4].

Using this lemma, we will prove a fundamental weighted isomorphism result for the Laplacian plus a constant. First we need to examine how the Laplacian behaves when conjugated by a power of $\rho$.

Lemma 3.2. Suppose $0<\alpha<1$ and $m+1+\alpha \leq k+\beta$. For $\varepsilon>0$, let $M_{\varepsilon}=\{x \in M: \rho(x) \leq \varepsilon\}$. For real numbers $\kappa$ and $s$,

$$
\rho^{-s}\left(\Delta_{g}+\kappa\right) \rho^{s}: \widetilde{\Lambda}_{m+2, \alpha}^{0}\left(M_{\varepsilon}\right) \rightarrow \Lambda_{m, \alpha}^{0}\left(M_{\varepsilon}\right)
$$

is an isomorphism whenever

$$
\left|s-\frac{n}{2}\right|^{2}<\frac{n^{2}}{4}+\kappa
$$

and $\varepsilon$ is sufficiently small.

Proof. Computing as in $\S 2$ of [GL], we obtain

$$
\begin{aligned}
\rho_{i j} & =\partial_{i} \partial_{j} \rho-\Gamma_{i j}^{k} \partial_{k} \rho \\
& =\partial_{i} \partial_{j} \rho-\left(\bar{\Gamma}_{i j}^{k}-\rho^{-1}\left(\delta_{i}^{k} \rho_{j}+\delta_{j}^{k} \rho_{i}-\bar{g}_{i j} \bar{\rho}^{k}\right)\right) \rho_{k} \\
& =\bar{\rho}_{i j}+\rho^{-1}\left(2 \rho_{i} \rho_{j}-\rho_{k} \bar{\rho}^{k} \bar{g}_{i j}\right),
\end{aligned}
$$

and so

$$
\rho_{i}{ }^{i}=\rho^{2} \bar{g}^{i j} \rho_{i j}=\rho^{2} \bar{\rho}_{i}{ }^{i}-(n-1) \rho \rho_{i} \bar{\rho}^{i} .
$$


Using this, we obtain

$$
\begin{aligned}
\rho^{-s}\left(\Delta_{g}+\kappa\right)\left(\rho^{s} u\right)= & \rho^{-s}\left(-\left(\rho^{s} u\right)_{; i}{ }^{i}+\kappa \rho^{s} u\right) \\
= & -u_{i}{ }^{i}-s \rho^{-1} \rho_{i}{ }^{i} u-2 s \rho^{-1} \rho^{i} u_{i}-s(s-1) \rho^{-2} \rho_{i} \rho^{i} u+\kappa u \\
= & -u_{i}{ }^{i}-s \rho \bar{\rho}_{i}{ }^{i} u+s(n-1) \rho_{i} \bar{\rho}^{i} u- \\
& \quad-2 s \rho \bar{\rho}^{i} u_{i}-s(s-1) \rho_{i} \bar{\rho}^{i} u+\kappa u \\
= & \left(\Delta_{g}+X+h\right) u
\end{aligned}
$$

where

$$
\begin{aligned}
X u & =-2 s \rho \bar{\rho}^{i} u_{i}, \\
h & =\kappa+s(n-s)|d \rho|_{\bar{g}}^{2}+s \rho \Delta_{\bar{g}} \rho .
\end{aligned}
$$

Since $|d \rho|_{\bar{g}}^{2} \rightarrow 1$ and $\rho \Delta_{\bar{g}} \rho \rightarrow 0$ at $\partial_{\infty} M$, it follows that $h$ is uniformly positive near $\partial_{\infty} M$ provided $\kappa+s(n-s)>0$, which is equivalent to (3.2). Choosing $\varepsilon$ sufficiently small, we see that $\rho^{-s}\left(\Delta_{g}+\kappa\right) \rho^{s}=\Delta_{g}+X+h$ satisfies the hypotheses of Lemma 3.1 on $M_{\varepsilon}$, and the lemma follows.

Proposition 3.3. Let $\kappa$ be a positive real number, $0<\alpha<1$, and $m+1+$ $\alpha \leq k+\beta$. Then

$$
\Delta_{g}+\kappa: \widetilde{\Lambda}_{m+2, \alpha}^{s}(M) \rightarrow \Lambda_{m, \alpha}^{s}(M)
$$

is an isomorphism whenever (3.2) holds.

Proof. Given $f \in \Lambda_{m, \alpha}^{s}(M)$, we need to show that there exists a unique $u \in \widetilde{\Lambda}_{m+2, \alpha}^{s}(M)$ such that

$$
\left(\Delta_{g}+\kappa\right) u=f .
$$

By the closed graph theorem, it follows then that $\left(\Delta_{g}+\kappa\right)$ has a bounded inverse.

The case $s=0$ is just a special case of Lemma 3.1. Consider next the case $s<0$. We can write $f=\rho^{s} b$ with $b \in \Lambda_{m, \alpha}^{0}(M)$. Then choosing $\varepsilon$ small enough that (3.1) is an isomorphism, we can find $v \in \widetilde{\Lambda}_{m+2, \alpha}^{0}\left(M_{\varepsilon}\right)$ satisfying $\rho^{-s}\left(\Delta_{g}+\kappa\right)\left(\rho^{s} v\right)=b$ on $M_{\varepsilon}$.

Let $\varphi \in C^{\infty}(\bar{M})$ be a function that is supported in $M_{\varepsilon}$ and identically equal to 1 in a neighborhood of $\partial_{\infty} M$. Then $\rho^{s} b-\left(\Delta_{g}+\kappa\right)\left(\varphi \rho^{s} v\right) \in \Lambda_{m, \alpha}^{0}(M)$ 
since it vanishes near $\partial_{\infty} M$; therefore by Lemma 3.1 again there exists $w \in \widetilde{\Lambda}_{m+2, \alpha}^{0}(M)$ satisfying

$$
\left(\Delta_{g}+\kappa\right) w=\rho^{s} b-\left(\Delta_{g}+\kappa\right)\left(\varphi \rho^{s} v\right) .
$$

It follows immediately that $u=w+\varphi \rho^{s} v$ solves (3.5), and since

$$
\widetilde{\Lambda}_{m+2, \alpha}^{0}(M) \subset \widetilde{\Lambda}_{m+2, \alpha}^{s}(M),
$$

we have $u \in \widetilde{\Lambda}_{m+2, \alpha}^{s}(M)$.

For uniqueness, suppose that $\left(\Delta_{g}+\kappa\right) u=0$ for some $u \in \widetilde{\Lambda}_{m+2, \alpha}^{s}(M)$. With $\varphi$ as above, it is easy to see that $h=\left(\Delta_{g}+\kappa\right)(\varphi u) \in \Lambda_{m, \alpha}^{0}\left(M_{\varepsilon}\right)$ because it vanishes near $\partial_{\infty} M$. Therefore, since (3.1) is an isomorphism for $s=0$ and $\varepsilon$ small, there is a unique $v \in \widetilde{\Lambda}_{m+2, \alpha}^{0}\left(M_{\varepsilon}\right)$ satisfying $\left(\Delta_{g}+\kappa\right) v=h$. But $\left(\Delta_{g}+\kappa\right)$ is also injective on $\widetilde{\Lambda}_{m+2, \alpha}^{s}\left(M_{\varepsilon}\right)$, so we must have $\varphi u=v \in$ $\widetilde{\Lambda}_{m+2, \alpha}^{0}\left(M_{\varepsilon}\right)$, so $u$ itself is in $\widetilde{\Lambda}_{m+2, \alpha}^{0}(M)$, and by injectivity of (3.4) in the case $s=0$ we conclude finally that $u=0$.

Now suppose $s>0$, and $f=\rho^{s} b \in \Lambda_{m, \alpha}^{s}(M)$. Since $\Lambda_{m, \alpha}^{s}(M) \subset$ $\Lambda_{m, \alpha}^{0}(M)$, by the $s=0$ case there exists $u \in \widetilde{\Lambda}_{m+2, \alpha}^{0}(M)$ satisfying (3.5). We need only show that $u \in \widetilde{\Lambda}_{m+2, \alpha}^{s}(M)$, for then injectivity on $\widetilde{\Lambda}_{m+2, \alpha}^{0}(M)$ automatically implies injectivity on the smaller space $\widetilde{\Lambda}_{m+2, \alpha}^{s}(M)$.

Choosing $\varepsilon$ small enough that (3.1) is an isomorphism, and letting $\varphi$ be a cutoff function as before, observe that $\rho^{-s}\left(\Delta_{g}+\kappa\right)(\varphi u) \in \widetilde{\Lambda}_{m, \alpha}^{0}\left(M_{\varepsilon}\right)$ since it agrees with $b=\rho^{-s} f$ near $\partial_{\infty} M$. Therefore by Lemma 3.2 there exists $v \in \widetilde{\Lambda}_{m+2, \alpha}^{0}\left(M_{\varepsilon}\right)$ satisfying

$$
\rho^{-s}\left(\Delta_{g}+\kappa\right) \rho^{s} v=\rho^{-s}\left(\Delta_{g}+\kappa\right)(\varphi u)
$$

on $M_{\varepsilon}$. By injectivity of (3.4) when $s=0$ it follows that $\varphi u=\rho^{s} v$, which completes the proof.

\section{The test function estimate.}

Armed with the isomorphism result of Proposition 3.3, we will show in this section how to use a power of a solution to the eigenvalue equation $\left(\Delta_{g}+n+1\right) u=0$ to construct a test function $\varphi$ satisfying $\left(\Delta_{g}-n^{2} / 4\right) \varphi \geq 0$. Throughout this section, we will assume only that $(M, g)$ is asymptotically hyperbolic of order at least $C^{2,0}$, without inner boundary. 
Proposition 4.1. Let $\rho$ be any smooth defining function. There exists a unique smooth, strictly positive function $u$ on $M$ satisfying

$$
\begin{gathered}
\left(\Delta_{g}+n+1\right) u=0, \\
u-\rho^{-1} \text { is bounded. }
\end{gathered}
$$

Proof. Let $\bar{g}=\rho^{2} g$. From (3.3) with $u=1$, we obtain

$$
\left(\Delta_{g}+n+1\right) \rho^{-1}=\bar{\rho}_{i}{ }^{i}+(n+1) \rho^{-1}\left(1-\rho_{i} \bar{\rho}^{i}\right) .
$$

The assumption that $\bar{g} \in C^{2,0}(\bar{M})$ guarantees that $\bar{\rho}_{i}{ }^{i} \in C^{0, \alpha}(\bar{M})$ for any $0<\alpha<1$. Moreoever, $1-\rho_{i} \bar{\rho}^{i} \in C^{1, \alpha}(\bar{M})$ and vanishes on $\partial_{\infty} M$ by the assumption of asymptotic hyperbolicity, so $\rho^{-1}\left(1-\rho_{i} \bar{\rho}^{i}\right) \in C^{0, \alpha}(\bar{M})$. Therefore, $\left(\Delta_{g}+n+1\right) \rho^{-1} \in C^{0, \alpha}(\bar{M}) \subset \Lambda_{0, \alpha}^{0}(M)$. It follows immediately from Proposition 3.3 that there exists a unique function $v \in \Lambda_{2, \alpha}^{0}(M)$ such that $u=\rho^{-1}+v$ satisfies (4.1) and (4.2).

Now, since $g$ is smooth in $M, u$ is smooth by local elliptic regularity. The strong maximum principle applied to (4.1) shows that $u$ cannot attain a non-positive interior minimum. Thus, since $u \rightarrow+\infty$ at $\partial_{\infty} M, u$ is strictly positive on $M$.

To prove uniqueness, let $v$ be the difference between two solutions; then $v$ is a bounded solution to $\left(\Delta_{g}+n+1\right) v=0$. Any bounded function is in $\Lambda_{0,0}^{0}(M)$ by definition, and then it follows from [GL, Proposition 3.4] that $v \in \Lambda_{2, \alpha}^{0}(M)$. Using the injectivity assertion of Proposition 3.3, we conclude that $v=0$.

We will take as our test function $\varphi=u^{-s}$, for a real power $s$ to be determined shortly. A straightforward computation gives

$$
\frac{\Delta_{g} \varphi}{\varphi}=-s \frac{\Delta_{g} u}{u}-s(s+1) \frac{|d u|_{g}^{2}}{u^{2}}=s(n+1)-s(s+1) \frac{|d u|_{g}^{2}}{u^{2}} .
$$

Since $u$ looks asymptotically like $\rho^{-1}$ near $\partial_{\infty} M$, it is reasonable to expect that, asymptotically,

$$
\frac{|d u|_{g}^{2}}{u^{2}} \sim \frac{\left|\rho^{-2} d \rho\right|_{g}^{2}}{\rho^{-2}}=\rho^{-2}|d \rho|_{g}^{2}=|d \rho|_{\bar{g}}^{2} \sim 1 .
$$

Therefore, near the boundary, $\Delta_{g} \varphi / \varphi$ approaches $s(n-s)$, so the best global estimate we can hope for is that $\Delta_{g} \varphi / \varphi \geq s(n-s)$. For $s>0,(4.4)$ shows 
that this is true precisely when $|d u|_{g}^{2} / u^{2} \leq 1$ on $M$. In that case $\varphi$ is a supersolution to $\Delta_{g}-s(n-s)$, so $s(n-s)$ is a lower bound for $\lambda_{0}(g)$. This bound is optimal when $s=n / 2$, in which case we get the lower bound $n^{2} / 4$ claimed in Theorem A.

This argument reduces the proof of Theorem A to the global gradient estimate

$$
|d u|_{g}^{2} \leq u^{2}
$$

for the eigenfunction $u$ of Proposition 4.1. We will accomplish this in two steps. First, in the next proposition, we will show that $|d u|_{g}^{2}-u^{2}$ is subharmonic when $g$ is Einstein, so it cannot take an interior maximum; then in the next section we will show that, under the hypotheses of Theorem A, $|d u|_{g}^{2}-u^{2} \leq 0$ on $\partial_{\infty} M$, so that (4.5) holds globally on $M$.

Proposition 4.2. Suppose $g$ is any Riemannian metric with $R i c_{g} \geq-n g$. Let $u$ be any solution to $\left(\Delta_{g}+n+1\right) u=0$. Then $|d u|_{g}^{2}-u^{2}$ is subharmonic.

Proof. A standard calculation using the Ricci identity and equation (4.1) shows that $u_{i j}{ }^{j}=(n+1) u_{i}+R_{i j} u^{j}$, and so

$$
\begin{aligned}
\Delta_{g}\left(|d u|_{g}^{2}-u^{2}\right) & =-\left(u_{i} u^{i}-u^{2}\right)_{; j}{ }^{j} \\
& =-2 u_{i j}{ }^{j} u^{i}-2 u_{i j} u^{i j}+2 u_{j}{ }^{j} u+2 u_{j} u^{j} \\
& =-2 n u_{j} u^{j}-2 R_{i j} u^{i} u^{j}-2 u_{i j} u^{i j}+2(n+1) u^{2} .
\end{aligned}
$$

Let $b=\nabla_{g}^{2} u+\frac{1}{n+1}\left(\Delta_{g} u\right) g$ denote the traceless Hessian of $u$. Then

$$
|b|_{g}^{2}=\left(u_{i j}-u g_{i j}\right)\left(u^{i j}-u g^{i j}\right)=u_{i j} u^{i j}-(n+1) u^{2} .
$$

Inserting this into (4.6), we get

$$
\Delta_{g}\left(|d u|_{g}^{2}-u^{2}\right)=-2\left\langle\left(R i c_{g}+n\right) d u, d u\right\rangle_{g}-2|b|_{g}^{2} \leq 0,
$$

which completes the proof.

\section{Boundary Asymptotics.}

In this section, we will compute the boundary asymptotics of the eigenfunction $u$ of Proposition 4.1 on an asymptotically hyperbolic Einstein manifold. The main result is Proposition 5.3, which gives the boundary values of $|d u|_{g}^{2}-u^{2}$. At the end of the section, we prove Theorem A. 
We assume in this section that $(M, g)$ is asymptotically hyperbolic of order $C^{3, \alpha}$, without inner boundary, and $g$ is Einstein.

Lemma 5.1. Let $\rho$ be any smooth defining function for $M$. Then there exists a defining function $r \in C^{2, \alpha}(\bar{M})$ such that

1. $r=\rho+O\left(\rho^{2}\right)$;

2. $\widetilde{g}=r^{2} g$ has a $C^{2, \alpha}$ extension to $\bar{M}$; and

3. $|d r|_{\bar{g}}^{2} \equiv 1$ in a neighborhood of $\partial_{\infty} M$.

Proof. Writing $\bar{g}=\rho^{2} g$ and $r=e^{v} \rho$, and computing as in the proof of Lemma 5.2 of [GL], we see that (3) is equivalent to

$$
2\langle d \rho, d v\rangle_{\bar{g}}+\rho|d v|_{\bar{g}}^{2}=\frac{1-|d \rho|_{\bar{g}}^{2}}{\rho} .
$$

If $v \in C^{2, \alpha}(\bar{M})$ is a solution to (5.1), then (2) and (3) will be satisfied, and (1) will follow if in addition $v=0$ on $\partial_{\infty} M$.

Now (5.1) is a non-characteristic first-order PDE for $v$, and can be solved easily in a neighborhood of $\partial_{\infty} M$ by Hamilton-Jacobi theory. However, since the standard treatments of Hamilton-Jacobi theory do not give Hölder regularity of the solution when the equation has Hölder coefficients, we will go through the construction in some detail.

Let $F: T^{*} \bar{M} \rightarrow \mathbf{R}$ denote the function

$$
F(x, \xi)=2\langle d \rho(x), \xi\rangle_{\bar{g}}+\rho(x)|\xi|_{\bar{g}}^{2}-\frac{1-|d \rho(x)|_{\bar{g}}^{2}}{\rho(x)}
$$

for $x \in \bar{M}, \xi \in T_{x}^{*} \bar{M}$. Solving (5.1) is equivalent to finding a function $u$ on $\bar{M}$ such that $F(x, d u(x)) \equiv 0$.

Since $\bar{g} \in C^{3, \alpha}(\bar{M})$ by hypothesis, the first two terms in $F$ are $C^{3, \alpha}$ functions on $T^{*} \bar{M}$. Since $1-|d \rho|_{\bar{g}}^{2}$ is in $C^{3, \alpha}(\bar{M})$ and vanishes on $\partial_{\infty} M$, it follows that $\left(1-|d \rho|_{\bar{g}}^{2}\right) / \rho \in C^{2, \alpha}(\bar{M})$, and hence $F \in C^{2, \alpha}\left(T^{*} \bar{M}\right)$. Thus the Hamiltonian vector field $X_{F}$ of $F$ is in $C^{1, \alpha}\left(T^{*} \bar{M}\right)$, and by Lemma A.1 in the Appendix, its flow $\varphi: \mathbf{R} \times T^{*} \bar{M} \rightarrow T^{*} \bar{M}$ is a $C^{1, \alpha}$ map wherever it is defined.

Write $b=\left(1-|d \rho|_{\bar{g}}^{2}\right) / \rho$, and let $\psi: \overline{\mathbf{R}^{+}} \times \partial_{\infty} M \rightarrow T^{*} \bar{M}$ be the $C^{1, \alpha}$ map

$$
\psi(t, x)=\varphi\left(t,\left(x, \frac{1}{2} b(x) d \rho(x)\right)\right) .
$$


In other words, $\psi$ is the flow-out by $X_{F}$ from the set $\left\{\left(x, \frac{1}{2} b(x) d \rho(x)\right) \in\right.$ $\left.T^{*} \bar{M}: x \in \partial_{\infty} M\right\}$. Since $F\left(x, \frac{1}{2} b(x) d \rho(x)\right)=0$ for $x \in \partial_{\infty} M$, a standard argument in symplectic geometry shows that the image of $\psi$ is a $C^{1, \alpha}$ Lagrangian submanifold-with-boundary of $T^{*} \bar{M}$ contained in $F^{-1}(0)$. It is easy to check that it is transverse to the fibers of $T^{*} \bar{M}$ along $\partial_{\infty} M$, and so near $\partial_{\infty} M$ it is the image of a closed 1-form $\omega \in C^{1, \alpha}\left(\bar{M}, T^{*} \bar{M}\right)$.

At least locally, $\omega=d v$ for some function $v \in C^{2, \alpha}(\bar{M})$. Since $\omega=\frac{1}{2} b d \rho$ along $\partial_{\infty} M$, any such function $v$ is locally constant on $\partial_{\infty} M$; if we require $v=0$ on $\partial_{\infty} M$, then $v$ is uniquely determined, and thus globally defined in a neighborhood of $\partial_{\infty} M$. Extending $v$ arbitrarily to all of $\bar{M}$ and setting $r=e^{v} \rho$ completes the proof of the lemma.

Lemma 5.2. With $u$ as in Proposition 4.1 and $r$ as in the preceding lemma,

$$
u=r^{-1}+r v
$$

where $v$ is a $C^{2, \alpha}$ function on $M$ such that $v$ and $|d v|_{g}$ have continuous extensions to $\bar{M}$, with

$$
v=\frac{1}{4 n(n-1)} \widehat{R} \quad \text { and } \quad|d v|_{g}=0 \quad \text { on } \partial_{\infty} M,
$$

where $\widehat{R}$ is the scalar curvature of $\widehat{g}=\left.r^{2} g\right|_{T \partial_{\infty} M}$.

Proof. Writing $\widetilde{g}=r^{2} g$, the formula for the transformation of the Ricci tensor under a conformal change of metric (cf., for example, [B, p. 59]) can be written

$$
R_{i j}=\widetilde{R}_{i j}+(n-1) r^{-1} \tilde{r}_{i j}+r^{-1} \tilde{r}_{k}{ }^{k} \widetilde{g}_{i j}-n r^{-2} r_{k} \tilde{r}^{k} \widetilde{g}_{i j},
$$

where quantities with tildes are computed with respect to $\widetilde{g}$. Using $r_{k} \tilde{r}^{k}=1$ and multiplying by $r$, near $\partial_{\infty} M$ the Einstein equation $R_{i j}=-n g_{i j}$ becomes

$$
r \widetilde{R}_{i j}+(n-1) \tilde{r}_{i j}+\tilde{r}_{k}^{k} \widetilde{g}_{i j}=0 .
$$

We will need two simple consequences of this formula. First, taking the trace with respect to $\widetilde{g}$, we get

$$
\tilde{r}_{i}^{i}=-\frac{1}{2 n} r \widetilde{R}
$$


Second, contracting (5.2) with $\tilde{r}^{i} \tilde{r}^{j}$ and using the fact that $\tilde{r}_{i j} \tilde{r}^{i} \tilde{r}^{j}=\frac{1}{2}\left(r_{i} \tilde{r}^{i}\right)_{; j} \tilde{r}^{j}=0$,

$$
\tilde{r}_{i}{ }^{i}=-r \widetilde{R}_{i j} \tilde{r}^{i} \tilde{r}^{j}
$$

These last two equations together imply

$$
\widetilde{R}_{i j} \tilde{r}^{i} \tilde{r}^{j}=\frac{1}{2 n} \widetilde{R}
$$

Next we will relate $\widetilde{R}$ with the scalar curvature $\widehat{R}$ of the boundary metric $\widehat{g}$. From (5.2) and (5.4) it follows that $\tilde{r}_{i j}=0$ on $\partial_{\infty} M$, so $\partial_{\infty} M=\{r=0\}$ is totally geodesic in the $\widetilde{g}$ metric. Therefore, at any point $x \in \partial_{\infty} M$, in terms of an orthonormal basis $\left\{X_{0}, X_{\alpha}, \alpha=1, \ldots, n\right\}$ for $T_{x} \bar{M}$ with $X_{0}$ normal to $\partial_{\infty} M$ and $X_{\alpha}$ tangent to $\partial_{\infty} M$, the curvature tensors of $\widetilde{g}$ and $\widehat{g}$ satisfy $\widetilde{R}_{\alpha \beta \gamma \delta}=\widehat{R}_{\alpha \beta \gamma \delta}$. Consequently the respective scalar curvatures satisfy

$$
\widetilde{R}=\widetilde{R}_{\alpha \beta}^{\alpha \beta}+\widetilde{R}_{\alpha 0}^{\alpha 0}+\widetilde{R}_{0 \beta}^{0 \beta}=\widehat{R}+2 \widetilde{R}_{00} .
$$

Since $|d r|_{\tilde{g}}=1, \widetilde{R}_{00}=\widetilde{R}_{i j} \tilde{r}^{i} \tilde{r}^{j}$ on $\partial_{\infty} M$, and so from (5.6) and (5.5) we conclude that

$$
\widehat{R}=\widetilde{R}-2 \widetilde{R}_{i j} \tilde{r}^{i} \tilde{r}^{j}=\frac{n-1}{n} \widetilde{R} \quad \text { on } \partial_{\infty} M .
$$

From (4.3), near $\partial_{\infty} M$ we have

$$
\left(\Delta_{g}+n+1\right) r^{-1}=\tilde{r}_{i}^{i}=-\frac{1}{2 n} r \widetilde{R} .
$$

Let $f \in C^{\infty}(\bar{M})$. Then a computation similar to (3.3) yields

$$
\left(\Delta_{g}+n+1\right)(r f)=2 n r f+r^{2}\left((n-3) \tilde{r}^{i} f_{i}-f \tilde{r}_{i}^{i}-r \tilde{f}_{i}^{i}\right) .
$$

From the last three equations, we conclude that, if $f$ is chosen so that $\left.f\right|_{\partial_{\infty} M}=\widehat{R}$

$$
\left(\Delta_{g}+n+1\right)\left(r^{-1}+\frac{1}{4 n(n-1)} r f\right)=r h,
$$

for some function $h \in C^{0, \alpha}(\bar{M})$ which vanishes on $\partial_{\infty} M$. If $0<\varepsilon \leq \alpha / 2$, it can be verified easily that $r h \in \Lambda_{0, \varepsilon}^{1+\varepsilon}(M)$, and so from Proposition 3.3 we conclude there is a unique function $w \in \Lambda_{2, \varepsilon}^{0}(M)$ such that $r^{1+\varepsilon} w \in \Lambda_{2, \varepsilon}^{1+\varepsilon}(M)$ satisfies

$$
\left(\Delta_{g}+n+1\right)\left(r^{-1}+\frac{1}{4 n(n-1)} r f+r^{1+\varepsilon} w\right)=0 .
$$


Since $r^{-1}-\rho^{-1}$ is bounded, by the uniqueness part of Proposition 4.1, we conclude that

$$
u=r^{-1}+\frac{1}{4 n(n-1)} r f+r^{1+\varepsilon} w .
$$

If we set $v=f /(4 n(n-1))+r^{\varepsilon} w$, it follows that

$$
\left.v\right|_{\partial_{\infty} M}=\left.\frac{1}{4 n(n-1)} f\right|_{\partial_{\infty} M}=\frac{1}{4 n(n-1)} \widehat{R}
$$

and

$$
|d v|_{g}=r|d v|_{\tilde{g}} \leq C\left(r|d f|_{\tilde{g}}+r^{1+\varepsilon}|d w|_{\tilde{g}}+|w| r^{\varepsilon}|d r|_{\tilde{g}}\right) \leq C^{\prime} r^{\varepsilon}
$$

This completes the proof.

Proposition 5.3. Suppose $\rho$ is any smooth defining function for $M$, and let $\widehat{g}=\left.\rho^{2} g\right|_{T \partial_{\infty} M}$. If $u$ is the solution to $\left(\Delta_{g}+n+1\right) u=0$ given by Proposition 4.1, then $|d u|_{g}^{2}-u^{2}$ has a continuous extension to $\bar{M}$, and is equal to $-\frac{1}{n(n-1)} \widehat{R}$ along $\partial_{\infty} M$.

Proof. Using the asymptotic expansion of $u$ given by the preceding lemma together with the fact that $|d r|_{g}^{2}=r^{2}$ near $\partial_{\infty} M$, we compute

$$
\begin{aligned}
|d u|_{g}^{2}-u^{2}= & \left|\left(-r^{-2}\right) d r+v d r+r d v\right|_{g}^{2}-\left(r^{-1}+r v\right)^{2} \\
= & r^{-4}|d r|_{g}^{2}+v^{2}|d r|_{g}^{2}+r^{2}|d v|_{g}^{2}-2 r^{-2} v|d r|_{g}^{2}-2 r^{-1}\langle d r, d v\rangle_{g} \\
& \quad+2 r v\langle d r, d v\rangle_{g}-r^{-2}-2 v-r^{2} v^{2} \\
= & -4 v-2 r^{-1}\langle d r, d v\rangle_{g}+O\left(r^{2}\right) .
\end{aligned}
$$

Since $|d v|_{g} \rightarrow 0$ at $\partial_{\infty} M$, this expression approaches $-4 v=-\frac{1}{n(n-1)} \widehat{R}$ at $\partial_{\infty} M$, which completes the proof.

Proof of Theorem A. If $g$ is as in the statement of the theorem, $\rho$ is any smooth defining function for $M$, and $u$ is as in Proposition 4.1, Proposition 5.3 shows that $|d u|_{g}^{2}-u^{2}$ approaches a negative constant times $\widehat{R}$ at $\partial_{\infty} M$. By the solution of the Yamabe problem [Sc], $\rho$ can be chosen so that $\widehat{R}$ is constant on $\partial_{\infty} M$, and the assumption that $y[\widehat{g}] \geq 0$ guarantees that this constant is non-negative. Since $|d u|_{g}^{2}-u^{2}$ is subharmonic by Proposition 4.2 and non-positive on $\partial_{\infty} M$, it follows from the maximum principle that $|d u|_{g}^{2} \leq u^{2}$ on all of $M$, and thus from (4.4) the positive function $\varphi=u^{-n / 2}$ 
satisfies $\Delta_{g} \varphi \geq\left(n^{2} / 4\right) \varphi$ on $M$, so the spectrum of $\Delta_{g}$ is bounded below by $n^{2} / 4$.

\section{Appendix: Hölder regularity for ODE's.}

The purpose of this appendix is to extend the standard regularity theory for ordinary differential equations to show that the flow of a vector field with Hölder coefficients has the same Hölder regularity as the coefficients. This is probably well-known to PDE experts, but I was unable to find it in the literature, so I have included a proof of the $C^{1, \alpha}$ case, which is all that is needed for this paper.

Lemma A.1. Let $V$ be a vector field on an open subset in $\mathbf{R}^{m}$, whose coefficient functions are of class $C^{1, \alpha}, 0<\alpha<1$. The solution $y(x, t)$ to the initial value problem

$$
\begin{aligned}
\frac{\partial y}{\partial t}(x, t) & =V(y(x, t)), \\
y(x, 0) & =x
\end{aligned}
$$

is a $C^{1, \alpha}$ function of $(x, t)$ where it is defined.

Proof. By classical ODE regularity theory, $y$ is a $C^{1}$ function of $(x, t)$. Since $\partial y^{i} / \partial t=V^{i} \circ y \in C^{1} \subset C^{0, \alpha}$, we need only show that $\partial y^{i} / \partial x^{j} \in C^{0, \alpha}$.

Differentiating (A.1) with respect to $x^{j}$ yields

$$
\frac{\partial^{2} y^{i}}{\partial x^{j} \partial t}(x, t)=\frac{\partial V^{i}}{\partial x^{k}}(y(x, t)) \frac{\partial y^{k}}{\partial x^{j}}(x, t) .
$$

Write $A_{k}^{i}(x, t)=\partial V^{i} / \partial x^{k}(y(x, t))$ and $u_{j}^{i}(x, t)=\partial y^{i} / \partial x^{j}(x, t)$. Since the composition of a $C^{1}$ function and a $C^{0, \alpha}$ function is in $C^{0, \alpha}, A_{k}^{i}$ is of class $C^{0, \alpha}$. Since $\partial^{2} y^{i} / \partial x^{j} \partial t$ is continuous, we can commute the second derivatives and conclude that $u_{j}^{i}$ satisfies the linear initial value problem

$$
\begin{aligned}
\frac{\partial u_{j}^{i}}{\partial t}(x, t) & =A_{k}^{i}(x, t) u_{j}^{k}(x, t), \\
u_{j}^{i}(x, 0) & =\delta_{j}^{i} .
\end{aligned}
$$

(The initial condition follows from differentiating (A.2) with respect to $x^{j}$.) 
For any fixed $x_{1}$ and $x_{2}$, define

$$
v_{j}^{i}(t)=u_{j}^{i}\left(x_{1}, t\right)-u_{j}^{i}\left(x_{2}, t\right) .
$$

Note that $v_{j}^{i}(0)=0$, and that the equation for $u_{j}^{i}$ implies

$$
\left|\frac{\partial v}{\partial t}(t)\right| \leq\|u\|_{L^{\infty}}\|A\|_{C^{0, \alpha}}\left|x_{1}-x_{2}\right|^{\alpha}+\|A\|_{L^{\infty}}|v(t)| .
$$

By one version of a standard ODE comparison theorem [H, Cor. III.4.3, p. 27], this implies that $|v(t)|$ is bounded by the solution to the IVP

$$
\begin{aligned}
y^{\prime}(t) & =\|A\|_{C^{0, \alpha}}\left(\|u\|_{L^{\infty}}\left|x_{1}-x_{2}\right|^{\alpha}+y(t)\right), \\
y(0) & =0
\end{aligned}
$$

which is

$$
y(t)=\left(\exp t\|A\|_{C^{0, \alpha}}-1\right)\|u\|_{L^{\infty}}\left|x_{1}-x_{2}\right|^{\alpha} .
$$

This yields the desired estimate.

Remark. This proof can easily be adapted to obtain higher Hölder regularity for the solution if $V \in C^{m, \alpha}, m>1$. However, as was pointed out to me by Curt McMullen, the analogous result fails for vector fields that are merely $C^{0, \alpha}$; in fact, there are vector fields with coefficients in the "Zygmund class", thus in $C^{0, \alpha}$ for every $0<\alpha<1$ and uniquely integrable, but whose solutions have arbitrarily bad Hölder exponents. See $[R]$ for further discussion.

\section{References.}

[B] A. L. Besse, "Einstein Manifolds," Springer-Verlag, New York, 1987.

[CY] S.-Y. Cheng and S.-T. Yau, Differential equations on Riemannian manifolds and their geometric applications, Comm. Pure Appl. Math. 28 (1975) 333354.

[FG] C. Fefferman and C. R. Graham, Conformal invariants, in "Elie Cartan et les Mathématiques d'aujourd'hui," Astérisque (1985) 95-116.

[GL] C. R. Graham and J. M. Lee, Einstein metrics with prescribed conformal infinity on the ball, Adv. Math. 87 (1991) 186-225. 
[H] P. Hartman, "Ordinary Differential Equations," Birkhäuser, Boston, 1982.

[LP] P. D. Lax and R. S. Phillips, The asymptotic distribution of lattice points in Euclidean and non-Euclidean spaces, J. Functional Analysis 46 (1982) 280-350.

[L] J. M. Lee, Fredholm operators and Einstein metrics on conformally compact manifolds, in preparation.

[M1] R. R. Mazzeo, The Hodge cohomology of a conformally compact metric, J. Differential Geom. 28 (1988) 171-185.

[M2] Elliptic theory of differential edge operators I, Comm. Partial Differential Equations 16 (1991) 1615-1664.

[M3] Unique continuation at infinity and embedded eigenvalues for asymptotically hyperbolic manifolds, Am. J. Math. 113 (1991) 25-45.

[P] H. Pedersen, Einstein metrics, spinning top motions and monopoles, Math. Ann. 274 (1986) 35-59.

[PS] R. Phillips and P. Sarnak, The Laplacian for domains in hyperbolic space and limit sets of Kleinian groups, Acta Math. 155 (1985) 173-241.

[R] H. M. Reimann, Ordinary differential equations and quasiconformal mappings, Invent. Math. 33 (1976) 247-270.

[Sc] R. Schoen, Conformal deformation of a Riemannian metric to constant scalar curvature, J. Differential Geometry 20 (1984) 479-495.

[SY] R. Schoen and S.-T. Yau, Conformally flat manifolds, Kleinian groups and scalar curvature, Invent. Math. 92 (1988) 47-71.

[Su] D. Sullivan, Related aspects of positivity in Riemannian geometry, J. Differential Geom. 25 (1987) 327-351.

UNIVERSITY OF WASHINGTON

DEPARTMENT OF Mathematics

Box 354350 Seattle, WA 98195-4350

E-MAIL: LEE@MATH.WASHINGTON.EDU

Received September 27th, 1994. 